\title{
Forming Subjective Representations of Subjective Representations: Evidence of a Subjective Status Bias
}

\author{
GUIDO PEETERS \\ Catholic University of Leuven \\ Leuven, Belgium
}

\begin{abstract}
In 3 studies and 2 pilot experiments, the author examined whether attending to the subjective status of mental representations would affect the ways humans view representational contents. The author found that simple drawing tasks were executed differently depending on whether or not the subject of the drawing was defined as a mental content (belief, imagination, perception). The results challenged particular lay epistemological concepts. They were partly accounted for by Gricean conversational rules (H. P. Grice, 1975), but the author postulated a subjective status bias to fully explain them. The discussion and recommendations for research center on the nature of this bias and relate it either to a tendency to conceive subjective representations as vague shadows of reality, or to an increased impact of the law of pregnance.
\end{abstract}

Key words: conversational logic, lay epistemology, subjective representation

ANY KNOWLEDGE OF THE WORLD IS SUBJECTIVE in the sense that it dependent upon a subject's mental activity, a principle that philosophers and psychologists have repeatedly stressed. For instance, Yates (1985) conceived the phenomenal world as a subjective model of the real world construed by the mental system in a way that enables the subject to survive through appropriate action.

In everyday life, however, people are naive realists. They assume that normal perceptual and mental functioning leads to objective knowledge that represents reality, as it is in itself, independent of the subject. This naive realism is manifested by the way we communicate. For instance, describing the grass, we

The author thanks Sara Demuzere and Judith Maier for assisting in data collection and three anonymous reviewers for their most helpful and constructive comments.

Address correspondence and requests for reprints to: Guido Peeters, Laboratory of Experimental Social Psychology, Tiensestraat 102, B-3000 Leuven, Belgium, guido.peeters@psy.kuleuven.be (e-mail). 
say: "It is green" rather than "It is known (e.g., perceived, told, believed,) to be green." Similar descriptions, from which any reference to a subject is omitted, are henceforth referred to as assertions. Descriptions in which a reference to the knowing subject is preserved are referred to as beliefs. Belief is a generic term applied to any description of reality in which the subjective representational status of the description is mentioned. Thus, it encompasses not only opinions or convictions such as "I think the grass is green," but also direct perceptions such as "I see the grass is green."

Our study deals with the question of whether perceivers accord differential informational value to assertions and beliefs. At first glance, one could expect that drawing a perceiver's attention to the belief status of a description would affect the perceiver's confidence in the validity of the description. Indeed, a belief can be an accurate representation of reality, but it can be biased or faked as well. However, reviewing studies on comprehension and on the processing of narratives (Gerrig, 1993; Gerrig \& Rapp, 2004; Gilbert, 1991) revealed ample evidence to suggest that humans are primarily set for accepting beliefs as being true. For example, Gilbert, Tafarodi, and Malone (1993) presented participants with manifestly false information and found that it was initially believed and, moreover, continued to be believed in conditions where mental load or time pressure prevented participants from "disbelieving" the false information. In the same vein, Gerrig (1989) found that truth judgments about obvious truisms were hindered by the availability of manifestly false belief contents from narrative contexts that cast doubt on the truisms. This effect persisted even if in a prior warning the objective truth of the truisms was affirmed. More recently, Rapp and Gerrig (2002) demonstrated that, when reading a narrative, one's knowledge of reality interacts with one's knowledge of the fictitious plot in a way that creates a reality-driven moment-by-moment experience. This experience preserves suspense even when the reader knows that the plot provides for a happy end. The notion is that the mental system is primarily set to believe rather than to disbelieve and it must invest extra effort to reject information as false. There seems no reason to expect perceivers to interpret beliefs differently from assertions, unless the perceivers have an indication that the belief deviates from reality.

At first glance, this conclusion seems challenged by a serendipitous observation from a study on linguistic information processing (Hendrickx, 1997). Hendrickx informed participants that a person "Jan" agreed with another person "Bert" that he, Jan, was taller than Bert. Then Hendrickx asked the participants who would really be the taller person. The participants answered "Jan," which confirmed not only Gerrig's (1993) and Gilbert's (1991) stand that perceivers are set for believing, but also Kruglanski's (1989) observation that perceivers rely on the consensus as a criterion of accuracy. However, when the same participants were asked to rate the accuracy of Jan's estimation, only 10 of 33 participants produced the expected complementary answer that Jan estimated his size correctly. Although Jan was right thinking he was taller than Bert, the majority as- 
sumed that he underestimated (1 participant) or overestimated (22 participants) his size.

This observation seems to contradict the primacy of belief acceptance unless some specification is added. For instance, humans may be assumed not to accept belief contents as perfectly accurate representations of reality, but as relatively accurate approximations of reality that enable one to act efficiently. Gerrig (1993) and Gilbert (1991) have specified that the unconditional acceptance of a statement as true is limited to the initial phase of achieving understanding of the statement. This initial acceptance functions as an onset hypothesis that is only preserved if it is not invalidated in the subsequent cognitive elaboration process. It is feasible that the rich social setting of Hendrickx's experiment involved uncontrolled cues that detracted from the credibility of the target persons. For example, participants may hold particular stereotypes that tall people overestimate their sizes. Hence, our first study was designed to try out a sober nonsocial setting. Our working hypothesis was that if the status of belief is accorded to a description of reality, it will affect the correspondence assumed between the description and the reality described (belief effect).

\section{STUDY 1}

\section{Method and Design}

\section{Participants and Procedure}

Three hundred and seventy-one Dutch speaking first-year psychology undergraduate students from the University of Leuven agreed to participate in an anonymous paper-and-pencil experiment on the optimal combination of text and picture. In collective sessions with about 60 participants supervised by a research assistant, and myself, the participants received a sheet with written instructions and a landscape-oriented frame. Following the instructions they then drew two lines within the frame that would best fit the caption under the frame. The captions varied across 16 conditions ( $\mathrm{C} 1$ to $\mathrm{C} 16)$ divided randomly among participants. All captions contained the same description that the upper line is longer than the lower line. Finally, I debriefed the participants and provided them with information about how they could obtain more information about the study. The appendix shows the full instructions used in the studies and Table 1 lists crucial elements.

\section{Design}

To check the generality of possible assertion-belief effects, and to explore their limits, the basic design involved two sets of conditions: (a) three assertion conditions, which were three variants of a control condition presenting the description in the caption as an assertion, and (b) 13 belief conditions, which were 
TABLE 1. Crucial Instruction Elements (Captions) and Results Based on 16 Conditions (C1-C16)

Condition $\quad M \quad S D \quad n \quad$ Dev.

\section{Assertion conditions}

C1 The upper line is longer. . .

$\begin{array}{llll}.70 & .08 & 21 & 0\end{array}$

C2 The upper line is surely longer. . .

$.72 \quad .08$

23

C3 It is sure that the upper line is longer. . .

$.69 \quad .08$

22

0

Covert belief conditions

C4 I think the upper line is longer. . .

C5 I am sure the upper line is longer. . .

$\begin{array}{llll}.58 & .11 & 22 & 2\end{array}$

C6 I see the upper line is longer. . .

$\begin{array}{llll}.68 & .12 & 23 & 2\end{array}$

C7 Kris thinks the upper line is longer. . .

$.66 \quad .13$

C8 Kris is sure the upper line is longer. . .

$.58 \quad .14$

C9 Kris sees the upper line is longer. . .

C10 Everybody thinks the upper line is longer. . .

C11 Everybody is sure the upper line is longer. . .

C12 Everybody sees the upper line is longer. . .

.67

Overt belief condition

C13 Kris says the upper line is longer.

C14 Kris says: "The upper line is longer. ."

C15 Everybody says the upper line is longer. . .

Note. Dev. $=$ deviants.

variants of an experimental condition presenting the description as a belief. The assertion conditions $\mathrm{C} 1-\mathrm{C} 3$ differed by the degree of certainty expressed $(\mathrm{C} 1 \mathrm{vs}$. $\mathrm{C} 2$ ) and by the sentence structure (C2 vs. C3). The belief conditions involved two sets of conditions referred to as the covert belief conditions and overt belief conditions. They could be regarded as two parallel experiments with the pooled assertion conditions as a common control condition.

Covert belief conditions. In nine conditions (C4-C12), the line descriptions were presented as cognitive and perceptual representations of one or more individuals. Similar representations constitute covert beliefs in that they are not accessible to others unless the individual communicates them. The conditions formed a $3 \times 3$ design involving the factors subject and modality. I manipulated the factor subject to control possible effects of consensus. It involved three levels: (a) the everybody conditions $(\mathrm{C} 10, \mathrm{C} 11, \mathrm{C} 12)$ in which the belief was presented as an object of consensus because it was shared by everybody, (b) the I conditions $(\mathrm{C} 4, \mathrm{C} 5, \mathrm{C} 6)$ in which the belief was attributed to a single person re- 
ferred to by the first person pronoun $I$, and (c) the Kris conditions (C7, C8, C9) in which the belief was attributed to a single person referred to by a common Flemish boy's and girl's name Kris. I manipulated the factor Modality because Hendrickx (1997) formulated beliefs using the expression "thinking that...," which could not only refer to the mental act of thinking but also function as an idiomatic expression of doubt. The idea of doubt included in the dual meanings could provide the cue required for turning belief into disbelief. Hence, to control for possible doubt effects, the factor modality involved three levels: (a) thinking conditions $(\mathrm{C} 4, \mathrm{C} 7, \mathrm{C} 10)$ in which the expression "thinking that" was used, (b) sure conditions $(\mathrm{C} 5, \mathrm{C} 8, \mathrm{C} 11)$ in which the belief was presented as a firm conviction by substituting "being sure that" for "thinking that," and (c) seeing conditions (C6, C9, C12) in which credibility of the belief was pursued by presenting it as a direct perception. There is consistent evidence from developmental and cross-cultural research suggesting that seeing expresses little, if any more, certainty than thinking (Schwanenflugel, Fabricius, \& Noyes, 1996; Schwanenflugel, Fabricius, Noyes, Bigler, \& Alexander, 1994; Schwanenflugel, Martin, \& Takahashi, 1999). However, as Schwanenflugel et al. (1996) noted, such studies focused on the pure mental act of thinking, excluding the idiomatic think that has been found to express more doubt, (e.g., guess; Moore \& Furrow, 1991).

Overt belief conditions. In conditions C13-C16, the belief was presented as an overt verbal statement for which one could, in principle, be held accountable. The overt belief differed from the assertion, as it was defined in the introduction, in that it involved not only an apparent assertion, but also an explicit reference to one or more subjects as authors of the assertion. The overt belief conditions formed a $2 \times 2$ design involving the factors Subject and Discourse. As in the covert belief conditions, the factor subject enabled me to control possible effects of consensus by presenting the belief either as one person's (Kris in conditions $\mathrm{C} 13, \mathrm{C} 14)$ or as everybody's (everybody in conditions C15, C16). The factor discourse enabled me to control the possible effects of sentence structure. As direct and indirect discourses differ considerably in Dutch, I contrasted indirect discourse conditions $(\mathrm{C} 13, \mathrm{C} 15)$ with direct discourse conditions $(\mathrm{C} 14, \mathrm{C} 16)$.

\section{Results}

All participants complied with the instructions. Although horizontal lines were not compulsory, only one participant drew two vertical lines. About $88 \%$ drew parallel lines resembling railroad tracks; the others (scattered across conditions) produced lines that touched or would touch soon if they were prolonged. A judge using a ruler measured the lines, and obtained a single index for each participant by dividing the length of the upper line by the sum of both line lengths. An index value of .50 indicated that both lines were equally long. The more the length of the upper line dominated, the more the score approached one, and the more the length of the lower line dominated, the more it 
approached zero. The reliability of the index was high, because the correlation between two independent judges across a sample of 21 participants was nearly perfect $(r>.99)$.

Table 1 shows mean index values and standard deviations for Study 1. In agreement with the caption (upper line longer than lower line), the majority of participants $(n=335)$ scored over .50. However, 36 participants contradicted the caption. They fell into two groups: 17 participants scoring below .50 (upper line shorter than lower line) and 19 participants scoring at .50 (both lines equally long). Because of the similar distributions of participants across conditions, I pooled the groups and handled them as a single group of 36 deviants. Table 1 shows the frequencies of deviants. The table shows that all of the deviants belonged to belief conditions, and none of them belonged to an assertion condition. Although this belief effect is statistically significant, because of the large number of nondeviants involved, the effect size $(E S)$-indicated by product moment (Cohen, 1977)—is rather small, $\chi^{2}(1, N=371)=8.63 p<.002$ (one-tailed), $E S=.15$.

\section{The Assertion Conditions}

Table 1 shows almost the same outcomes for the three assertion conditions and, after performing $t$ tests, I found no statistically significant difference. Thus, it did not matter whether the assertion was a straightforward affirmation (C1) or an affirmation qualified as sure using either an adverb (C2) or a compound sentence structure (C3) that resembled the sentence structure used in the belief conditions. Hence, in subsequent analyses, I pooled the three conditions into a single assertion control condition. The overall mean was .70, the standard deviation (.08) was rather small, and there were no deviants.

\section{The Covert Belief Conditions}

Table 1 shows that each covert belief condition yielded at least one deviant and a lower mean index value than in any of the assertion conditions. Table 2

TABLE 2. Mean Index Values for Covert and Overt Belief Conditions

\begin{tabular}{lcccccc}
\hline \multirow{2}{*}{ Subject } & \multicolumn{3}{c}{ Modality } & & \multicolumn{2}{c}{ Discourse } \\
\cline { 2 - 4 } \cline { 6 - 6 } & Thinking & Being Sure & Seeing & & Indirect & Direct \\
\hline I & .58 & .68 & .66 & & .66 \\
Kris & .58 & .66 & .66 & & .64 & .62 \\
Everybody & .53 & .67 & .68 & & .59 & .62 \\
& & & & & & \\
\hline
\end{tabular}


shows the index values. The index values were analyzed with a $3 \times 3$ type analysis of variance (ANOVA; Subject $\times$ Modality) with the pooled assertion control conditions added as an isolated hanging condition. The difference between the pooled assertion conditions $(M=.70)$ and the pooled covert belief conditions $(M$ $=.63)$ was statistically significant, $F(1,268)=19.33, p<.00002, M S E=0.01$, $E S=.26$ approaching conventional middle size (.30 according to Cohen, 1977). This effect persisted when deviants were removed from the analysis, $F(1,239)=$ $11.21, p<.001, M S E=0.01, E S=.21$. The effect of subject was not statistically significant, $(F<1)$, neither was the interaction between subject and modality, $(F=1)$. Apparently, it did not matter whether a belief was presented as everybody's belief or as the belief of one person. Thus, we obtained no evidence of a consensus effect. Moreover, the distribution of deviants across subject conditions (7, 6 and 18 in the I, Kris, and everybody conditions, respectively) suggested a reverse consensus effect as if being shared by everybody detracted from the belief's credibility.

There was a statistically significant main effect of modality, $F(2,268)=$ $20.95, p<.00001, M S E=0.01, E S=.36$. This effect could be because of a proportionally high number of deviants in the thinking conditions that keep down the average index. However, the effect persisted when deviants were removed from the analysis, $F(2,239)=19.86, p<.00002, M S E=0.01, E S=.37$. As explained earlier, the expression thinking that may have communicated doubt. This doubt may not only account for the relatively high number of deviants, but also for keeping nondeviant differences as small as possible. Thus, if the obtained belief effect is not a result of doubt, it should also be obtained in the sure and seeing conditions where beliefs are characterized by high certainty. The means in the separate sure and seeing conditions were nearly identical indicating that similar informational value was accorded to a conviction or sure belief as to a perception. The pooled mean for sure and seeing $(M=.67)$ was only slightly lower than the mean of the pooled assertion conditions $(M=.70)$, but the difference was statistically significant $F(1,268)=4.16, p<.05, M S E=0.01, E S=.12$. This significant difference indicates that the belief effect may not be a trivial effect of doubt communicated by the expression thinking that. However, it should be noted that when deviants were removed from the analysis, the difference was not statistically significant, though still in the same direction, $M=.69 F(1,239)=$ $0.78, p<.38, M S E=0.01, E S=.06$.

\section{The Overt Belief Conditions}

As shown in Table 1, in the overt belief conditions, mean index values were systematically lower than they were in the assertion conditions. The index values are also shown in Table 2 . They were subjected to a $2 \times 2$ ANOVA with the pooled assertion control condition added as an isolated hanging condition. The difference between pooled overt belief conditions $(M=.62)$ and pooled assertion conditions 
$(M=.70)$ was statistically significant and middle-sized, $F(1,154)=19.00, p<$ $.00003, M S E=0.01, E S=.33$. The difference remained statistically significant when deviants were removed from the analysis, $F(1,146)=12.94, p<.0005$, $M S E=0.001, E S=.29$. Thus, the belief effect persisted even when the belief was presented as an overt utterance.

The effect of direct versus indirect discourse was not statistically significant $(F<1)$ and neither was the interaction between discourse and subject $(F<1)$. The only statistically significant effect was the main effect of subject, $F(1,154)=$ $4.60, p<.04, M S E=0.01, E S=.03$, which was opposed to the effect that could be expected from consensus information. Indeed, one could expect that the greater length of the upper line would be most salient in conditions where everybody agrees on it. However, as shown in Table 2, index values were higher in the Kris condition than in the everybody condition. It should be noted that the difference was not statistically significant if deviants were removed from the analysis, $F(1,146)=0.587, p<.45, M S E=0.01$, but still, the expected consensus effect was not observed. Moreover, as in the covert belief conditions, the consensus effect was contradicted in that much more deviants $(n=7)$ belonged to the everybody conditions than to the Kris conditions $(n=1)$.

\section{Discussion}

Altogether, the data provided strong support for our hypothesis that the status of belief accorded to a description of particular facts affects the consistency assumed between the description and the facts. Apparently, the results suggest two possible belief effects henceforth referred to as the weak effect and the strong effect. The weak effect reduces only the saliency of the consistency assumed between the description and the facts without turning into inconsistency. The strong effect involves manifest inconsistencies exemplified by the drawings of the deviants.

The question arises whether the weak and strong belief effects are on par with each other. For instance, the deviants may not have taken the task seriously. Although this idea is highly questionable (i.e., Why would this occur exclusively in belief conditions?) it is advisable to consider the weak effect apart from the strong effect. Thereby, one may wonder if there is sufficient evidence of a belief effect if the strong effect is ignored and only the weak effect is considered.

Apparently, ignoring the strong effect, sufficient evidence of a belief effect is left by the weak effect. Covert as well as overt condition sets showed statistically significant belief effects after the deviants were removed from the analyses. The belief effect obtained in covert belief conditions was no longer statistically significant when not only data produced by deviants but also data from thinking conditions were omitted from the analysis. This could mean that the weak belief effect is restricted to beliefs expressed in a way that might suggest some doubt. However, this restriction was contradicted by the statistically significant weak 
belief effect in the overt belief conditions. Moreover, combining the statistically nonsignificant outcome from the covert belief conditions $(E S=.06)$ with the statistically significant outcome from the overt belief conditions $(E S=.29)$ yielded a statistically significant weak effect, $Z c=3.11, p<.001$ (one-tailed Stouffer Combined Test; Wolf, 1986). Thus, the weak belief effect persists even when the belief is formulated in a way excluding doubt. Hence, it seems warranted to conclude that there is a robust belief effect. It may be moderated by stressing the believer's confidence, or by grounding the belief on perception, and it may be separated into defined strong and weak effects.

\section{Some Epistemological Implications}

The present outcomes have epistemological implications for the theories that stress the primacy of belief acceptance in the comprehension process (Gerrig, 1993; Gilbert, 1991). For the data to fit the theories, we assumed that our experimental belief conditions involved cues that could have informed participants that the beliefs in question might not be accurate. A similar cue for doubt may have been provided by the expression thinking that, which was confirmed by the outstanding belief effect in the thinking conditions. However, doubt cannot explain the statistically significant belief effects observed when no doubt was expressed.

The belief effect was not reduced, but rather increased, when the belief was presented as a belief shared by everybody. This challenges the consensus principle that stresses consensus as a criterion of accuracy (Kruglanski, 1989). Apparently, mentioning the self-evident subjective status of a description as a product of cognitive or perceptual activity suffices to affect the informational value accorded to the description.

Nevertheless, a possible explanation of the observed belief effects that would not detract, either from the primacy of belief acceptance or from the consensus principle, may be available in Grice's (1975) logic of conversation.

Grice's concepts belong to the philosophy of language. However, they have appealed to not only to linguists (Levinson, 2000), but also students of social communication (Hilton, 1990, 1991, 1995; Schwarz, 1996, 1998). According to Grice (1975), communication involves an implicit contract between sender and receiver in accordance with a set of maxims of conversation, one of which is the maxim of quantity. It implies that the sender is expected to make the message as informative as is required for the purposes of the information exchange and not more so.

An analogous rule is the principle of optimal redundancy derived by Peeters (1977) from a critical examination of Heider's (1958) cognitive consistency concepts regarding the economy of interpretation. Heider's concepts imply that messages are interpreted to achieve maximal redundancy of the message contents. For that reason, the sentence "John went to the ball and he kicked the leather ob- 
ject" would communicate a football scene rather than a ballroom scene with a leather jacket being kicked away from the dance-floor. However, according to the principle of optimal redundancy, messages are interpreted to achieve optimal redundancy rather than maximal redundancy. If the level of optimal redundancy is exceeded (over-redundancy) the interpretation of the message would be adjusted to have the optimal level restored. For instance, the term warm refers primarily to the physical temperature of an object as in warm water. However if warm is used to describe an object that is warm by its nature, a secondary meaning may be called forth as in warm fire where warm may communicate the comfortable character of the fire rather than temperature.

Our participants may have conceived their task as the production of a picture with caption, as in an illustrated book. Drawing the upper line longer than the lower line, consistent with the caption, may have involved over-redundancy that was reduced by decreasing, or even reversing, the contrast between the line lengths.

A trouble with this explanation is that it does not explain why participants would feel more urged to reduce over-redundancy in the belief conditions than in the assertion conditions. A possible answer may be provided by another maxim of Grice (1975): the maxim of relation. Grice states that all information communicated is expected to be relevant. Hence, over-redundancy may be disregarded if it is perceived as a consequence of a relevant aspect of the communication such as teaching purposes or the necessity to avoid miscommunication (Hilton, 1995; Peeters, 1977). For example, the reader of a criminal story may read about various crimes committed by a criminal without regard for over-redundancy created in that each new crime reported communicates again that the criminal is a criminal. The over-redundancy would be set aside as an irrelevant consequence of reporting relevant information. Hence, in the assertion conditions, over-redundancy may be set aside as an irrelevant consequence of relevant information communicated by the caption. For instance, the caption may inform the reader that the issue at stake concerns the relative length of the lines and not some other aspect of the drawing.

Also in the belief conditions, the captions focus attention on the relative line lengths but they add that that the difference between the lines is perceived and believed. Such additional information may only be relevant if it is not too obvious from the picture that the upper line is indeed the longer one. Therefore, participants may have felt urged to provide for relevance by reducing manifest agreement with the picture, even to the point that the picture contradicted the caption. Such participants assigned relevance to the caption by creating a message that communicated a misperception or a false belief.

In the everybody conditions, the urge for creating relevance may have been strong enough to overrule the possible consensus effect. However, in conditions where the belief was presented as a specific person's belief, the urge for creating relevance may have been weaker. Indeed, participants could assign relevance to the information that a person whose name is Kris, or the partici- 
pant himself or herself, was part of the scene. The fact that the relevant information involved high redundancy does not necessarily urge a reduction of over-redundancy; the over-redundancy could be ignored as an irrelevant sideeffect of a relevant communication.

Because of the present rationale, we could also expect that, in the everybody conditions, the belief effect would be reduced if the everybody statement is integrated into more informative communication. In such a case, the belief effect may not only be reduced from over-redundancy's yield to relevance, but also because there may be room for the consensus effect. Considering that the requirement of a more informative communication may be met by the Kris conditions, we decided to have a partial replication of Study 1 that included a condition in which the caption had more information than either the somebody or the Kris condition. I hypothesized that (a) the belief effect obtained in Study 1 would be replicated, and (b) the belief effect would be reduced in the condition with the most informative caption.

\section{STUDY 2}

\section{Method and Design}

\section{Participants and Procedure}

I randomly drew 137 volunteers from a new generation of Leuven first-year psychology undergraduate students that could not have participated in Study 1. In a collective session, I randomly divided them across four conditions and presented them with the same line drawing task as in Study 1 (see Appendix).

\section{Conditions}

The captions for each condition are shown in the Appendix and in Table 2. To avoid confusion with Study 1, we numbered the captions consecutively across studies. C17 is the control condition assertion, which was an exact replication of $\mathrm{C} 1$. There were three experimental belief conditions. C18 (Kris condition) and $\mathrm{C} 19$ (everybody condition) were exact replications of $\mathrm{C} 13$ and $\mathrm{C} 15$, being conditions in which the description of the lines was presented as an overtly expressed belief of respectively one person (Kris) and many people (everybody). C20 was a new condition in which the caption was assumed to be more informative than in the two other belief conditions. To be able to relate possible effects of $\mathrm{C} 20$ to mere informativeness, we attempted to increase informativeness in C20 relative to $\mathrm{C} 18$ and $\mathrm{C} 19$ without introducing qualitatively novel information contents. Instead, the caption in C20 repeated the captions from C18 and C19 and added extra information by relating them to each other and telling the participants that Kris is not an exceptional person. 


\section{Results and Discussion}

Mean index values (with standard deviations) and numbers of deviants are shown in Table 3 . I subjected index values (see Table 4 ) to a $2 \times 2$ ANOVA with one factor contrasting conditions with references to Kris (C18 and C20) versus conditions without (C17 and $\mathrm{C} 19)$, and the other factor contrasting conditions with references to everybody (C19 and C20) versus conditions without (C17 and C18). The only statistically significant effect was a main effect that was consistent with the reverse consensus effect from Study 1. As shown in Table 4, I obtained lower index values when the description of the lines was presented as everybody's belief than when it was not, $F(1,133)=8.55, p<.004, M S E=0.01$, $E S=.25$.

To test our hypothesis that the belief effect would be replicated, I compared the assertion condition, $\mathrm{C} 17(M=.66)$, with the pooled belief conditions (C18, $\mathrm{C} 19$, and $\mathrm{C} 20 ; M=.62)$. Although the effect was rather small, it was statistically significant $F(1,133)=4.15, p<.04, M S E=0.01, E S=.17$. Thus, consistent with our hypothesis, the upper line was drawn proportionally longer in the as-

TABLE 3. Crucial Instruction Elements (Captions) and Results Based on Four Conditions (C17-C20)

\begin{tabular}{lllll}
\hline \hline Condition & $M$ & $S D$ & $n$ & Dev. \\
\hline Assertion conditions & & & & \\
C17 The upper line is longer. . & .66 & .09 & 36 & 1 \\
& & & & \\
Covert belief conditions & & & & \\
C18 Kris says the upper line is longer. . & .65 & .11 & 33 & 2 \\
C19 Everybody says the upper line is longer. . & .62 & .11 & 34 & 4 \\
C20 Like everybody else, Kris says. . & .59 & .10 & 34 & 8
\end{tabular}

Note. Dev. $=$ deviants.

TABLE 4. Mean Index Values Based on Conditions With and Without Reference to Everybody and Kris

$\begin{array}{lll}\text { Condition } & \text { With Kris } & \text { Without Kris }\end{array}$

$\begin{array}{lll}\text { Without Everybody } & .65 & .66 \\ \text { With Everbody } & .59 & .62\end{array}$


sertion condition than in the belief conditions. Also consistent with our hypothesis, there was only one deviant in the assertion condition versus 14 in the belief condition, $\chi^{2}(1, N=137)=2.304, p<.07$ (one-tailed test with correction for continuity), $E S=.16$ (effect size slightly higher than in Study 1).

When deviants were removed from the analysis, the difference between the assertion and belief conditions ( $M=.67$ and .64 , respectively) still pointed in the same direction but was not statistically significant $F(1,118)=1.57, p<.22, M S E$ $=0.01, E S=.11$. However, the belief effect was statistically significant in a metaanalysis combining the present outcome with outcomes from Study 1. It was also statistically significant when the deviants were removed, and also in the three thinking conditions $(\mathrm{C} 4, \mathrm{C} 7$, and $\mathrm{C} 10)$, which were the conditions with the largest belief effects, $Z c=3.26, p<.0006$ (one-tailed Stouffer Combined Test of the present effect, $E S=.11$, with effects from Study $1, E S=.06$ and .29 ; see Wolf, 1986). Such results validate the weak belief effect and confirm that it holds even for firm beliefs that involve no manifest doubt.

Our hypothesis that the belief effect would be reduced by the increased informativeness of the caption was not confirmed. Although the amount of information conveyed through the caption in $\mathrm{C} 20$ exceeded that of the captions in $\mathrm{C} 18$ and $\mathrm{C} 19$, the belief effect was not reduced. On the contrary, C20 yielded the lowest mean index and the highest number of deviants, lending no support to the theory that increasing the informative value of the caption would suspend the need to reduce over-redundancy. Therefore, we are left with the question of why overredundancy is avoided more in belief than in assertion conditions.

Continuing with the logic of conversation, a possible answer is that, in belief conditions, participants make sense of the picture with caption by conceiving the whole as a communication about false belief or misperception. However, in this way, we may explain the strong belief effect produced by the deviants but not the statistically significant weak belief effect. Hence, we hypothesize the existence of a primitive belief effect that requires no further explanation. This would mean that perceivers assume that mental representations of reality differ in a systematic way from reality simply by virtue of their mental representational status. I tested this hypothesis in Study 3 by no longer presenting the line drawing task as the production of a picture that should fit a caption.

\section{Study 3}

\section{Method and Design}

\section{Participants and Procedure}

I randomly drew another sample of 165 participants from the same population as in Study 2 and, in a collective session, I presented them with the same line drawing task. However, the task was no longer presented as the production of a picture 
with caption. Instead, the experimenter asked for simple drawings to be used in a future study (see Appendix). I randomly divided participants across five conditions.

\section{Design}

The experiment involved two main conditions that corresponded to the assertion and belief conditions from Studies 1 and 2. The experimenter asked for a drawing that would fit the description. However, to prevent the effects of avoiding over-redundancy, it was no longer implied that the picture and the description formed together a conventional picture with text message. Instead, I formatted the description as part of a request rather than as a declarative statement about reality. For that reason the terms assertion and belief were no longer appropriate to characterize conditions and I replaced them with factual representation and subjective representation, respectively. The instructions used to implement the factual representation and subjective representation conditions are presented in the Appendix and briefly in Table 5. For explorative purposes, and analogous to the belief conditions in previous studies, four parallel subjective representation conditions (C22-C25) were used and formed a $2 \times 2$ design that involved the factors subject and modality. Subject contrasted conditions in which participants were requested to draw the lines as people in general may represent them in their imagination (people conditions C22 and C23) against conditions in which they were asked to draw them as they themselves would represent them in their own imagination (self conditions $\mathrm{C} 24$ and C25). Modality involved a second-order assertion or belief manipulation. In the assertion conditions (C22 and C24), I asked participants to only draw the lines as they were imagined by subjects (people or themselves), while in the belief condition ( $\mathrm{C} 23$ and $\mathrm{C} 25)$ they were asked to draw

TABLE 5. Crucial Instruction Elements (Direct Requests) and Results Based on Four Conditions (C21-C25)

\begin{tabular}{lllll} 
Condition & $M$ & $S D$ & $n$ & Dev. \\
\hline $\begin{array}{l}\text { Factual representation condition } \\
\text { C21 upper line longer than lower line }\end{array}$ & .69 & .10 & 35 & 0 \\
Subjective representation conditions & & & & \\
Lines drawn as. . & & & & \\
C22 people imagine them & .6 & .07 & 33 & 0 \\
C23 you think people imagine them & .61 & .07 & 33 & 1 \\
C24 you imagine them & .67 & .07 & 30 & 0 \\
C25 you think you imagine them & .67 & .08 & 34 & 0
\end{tabular}

Note. Dev. $=$ deviants. 
them in the way they thought that subjects (people or themselves) would represent them in their imagination.

\section{Results and Discussion}

I analyzed the index data (see Table 5 and 6) with a $2 \times 2$ type ANOVA (Subject $\times$ Modality) with factual representation added as an isolated hanging condition. The difference between the factual representation and pooled subjective representation conditions was statistically significant, $F(1,160)=6.48, p<.02$, $M S E=0.01, E S=.20$. Such results pointed in the predicted direction across the four subjective representation conditions, which supports my hypothesis that subjective representations of reality are conceived differently from the way reality itself is. Specifically, reality seems conceived as more prominently featured than subjective representations of reality.

The ANOVA revealed a statistically significant main effect only for the factor subject, an indication that participants saw their own imagination more in agreement with reality than the imagination of people in general, $F(1,160)=$ $6.57, p<.02, M S E=0.01, E S=.20$. This outcome was again inconsistent with a possible epistemological consensus effect according to which subjective representations of people in general would fit reality better than a single individual's. However, it fits a possible self-enhancement or superiority bias (e.g., Hoorens, 1993) according to which participants may assume that their own representations fit reality better than do the representations others may form.

Finally, a noteworthy outcome was the nearly complete absence of deviants. There was only one deviant among 165 participants, which is less than $1 \%$ compared with $10 \%$ in Study 1 and $11 \%$ in Study 2 . Because the participants of Studies 2 and 3 were randomly drawn from the same population, the experiments can be considered two conditions of a single experiment and it makes sense to test the significance of the difference between the numbers of deviants. The difference was statistically significant, $\chi^{2}(1, N=302)=15.95, p<.00006, E S=.23$. In fact, the deviant rate of Study 3 also differs significantly from that of Study 1 , $\chi^{2}(1, N=536)=14.71, p=0.00013, E S=.17$. Only in situations where participants had to produce a picture with an appropriate combination of the picture

TABLE 6. Mean Index Values Based on the Factors Subject and Modality

Modality

Subject

Assertion

Belief

People

Self (you)
.66

.67
.61

.67 
and the given caption did, a significant proportion of the participants produced a picture that deviated manifestly from the description in the caption. Such a result suggests that, in agreement with the logic of conversation, the strong belief effects produced by the deviants reflected a tendency to avoid over-redundancy by conceiving the picture with caption as a communication about misperception or false belief. However, the statistically significant weak belief effect, produced by the nondeviant majority, still requires an explanation.

\section{General Discussion}

The presented data suggest the existence of belief effects in the sense that awareness of the subjective status of a representation of reality affects the way the representation is conceived. An observed strong belief effect suggests that people assume subjective representations of reality to deviate from reality. However, this effect was bound to particular conditions that suggest that the effect serves communicative purposes. In agreement with the Gricean logic of conversation, the effect could be related to optimal message redundancy, which requires that the message provides no more information than necessary and that each message component contributes significantly to the message content.

In addition to the strong belief effect, I obtained a persistent weak belief effect. It did not involve manifest inconsistencies between subjective representations and reality but subjective representations seemed less prominently featured than was reality. One could wonder whether the logic of conversation could not account also for the weak belief effect. For instance, participants may have aimed at optimal communication redundancy by reducing the salience of the agreement between caption and picture without breaking the agreement. It might be so in Studies 1 and 2, where the experimental line drawing task was presented as the construction of a communication. However, the effect persisted in Study 3, where participants were not instructed to construe a communication.

The present outcomes may bear upon a long-lasting philosophical controversy regarding propositional attitudes (for a review, see McKay, 2000). Propositional attitudes are expressed in sentences as the ones used in the belief conditions (e.g., Kris thinks that... I am sure that...). Consistent elaboration of their semantics has raised problems that reach beyond the scope of this article. However, there may be some ground in common with philosophers' thinking in the search for solutions. For instance, the present distinction between fact and subjective representation is reminiscent of a similar distinction advanced by Gottlob Frege (1892) in order to deal with the problems. An alternative approach of the problems, which was initiated by Bertrand Russell (1912), has led present-day philosophers, among who are McKay, to expect solutions from pragmatic theories such as Grice's (1975) logic of conversation at stake in the present study. However, according to McKinsey (1999), Russellians have failed to use the Gricean concepts. Apparently, McKay and McKinsey stand up for a Gricean ap- 
proach of propositional attitudes. The present studies fit into a similar approach. They may not provide a solution for the semantic problems philosophers have raised, but they do show that Gricean concepts shed light on the processing of propositional attitudes.

In the interpretation of the results, the logic of conversation was linked exclusively to the belief conditions where it accounted for the so-called strong effects. One could object that the logic of conversation may apply beyond the belief conditions and account for the weak effects in Study 3. Specifically, logic of conversation may have ruled the participants' interpretation of the instructions provided by the experimenter (Hilton, 1995). When the experimenter asked to draw the upper line longer than the lower line, and specified that lines should be drawn as if they are represented in imagination, participants may have felt urged to make sense of that instruction by deviating slightly from the way they would normally draw the lines. However, one could expect some participants to draw the upper line proportionally shorter, and others to draw it proportionally longer, than they normally would. This should have resulted in the same means, but larger standard deviations, in the subjective representation conditions than in the factual representation conditions. However, the data in Table 5 show that the standard deviations in the subjective representation conditions are not larger but rather smaller than in the factual representation conditions. Apparently, evidence for the logic of conversation is limited to belief conditions. Hence, it seems warranted to conclude that there exists what may be called a subjective status bias to which humans are inclined to conceive subjective representations differently from how they conceive reality. Future researchers should test the generality of this bias and specify its nature.

\section{Exploring the Subjective Status Bias}

The present experimental observations, as well as common sense, suggest the following shadow of reality hypothesis.

\section{The Shadow of Reality Hypothesis}

Possibly the subjective status bias boils down to a tendency to view mental representations as pale, more or less blurred, imitations or shadows of reality. Any description or representation is a product of mental activity and so involves a subjective status. However, the subjective status is ignored unless it is brought to a person's attention. This may have happened in Hendrickx's (1997) study. In agreement with the shadow of reality hypothesis, Hendrickx's data suggest that even if subjective mental representations are assumed to be correct, they are not yet assumed to be exactly correct. They are viewed only as approximations of reality that may be sufficient to program efficient action and to safeguard a coherent world view. 
At a first glance, this description of mental representations matches Gerrig's (1993) description of readers' moment-by-moment interpretation of narratives. In agreement with Gilbert (1991), Gerrig stressed that the reader's experience of nonfiction is primarily not differentiated from fiction. Nevertheless, he also reviewed convincing evidence that fiction-based representations tend to be incomplete as compared with veridical representations. For example, when reading a restaurant scene, readers automatically add realistic details that are not explicitly communicated. However, they systematically omit particular essential details such as the cutlery used by the guests. In point of fact, details are only inferred as far as they ensure local coherence (McKoon \& Radcliff, 1992).

The latter omissions are in line with the idea that mental representations are handled as approximations rather than full-fledged representations of reality. At a first glance, this fits the shadow of reality hypothesis. However, the specification that the omissions serve to achieve coherence casts doubt on the hypothesis. Indeed, coherence is a feature at odds with the conception of the mental representation as a vague shadow or a pale imitation of reality. In anticipation of more extensive research dealing with the matter, I ran two pilot studies.

\section{Pilot Study 1: Exploring the Shadow of Reality Hypothesis}

According to the shadow of reality hypothesis, subjective representations involve a reduction of reality that is not restricted to the omission of particular details but affects the whole. Hence one might expect mental representations to be portrayed smaller than representations of reality. In my experiments, this would have resulted in smaller drawings in the belief and subjective representation conditions than in the assertion and factual representation conditions. However, careful examination of absolute line lengths did not yield supportive evidence. Apparently, the length of the (longer) upper line was set by the size of the frame and differences between conditions were achieved by varying the length of the (shorter) lower line.

To avoid possible effects due to the constraints of a drawing task, I used a different paradigm than the one used in Studies 1-3. During a college lecture, $65 \mathrm{fe}-$ male first-year undergraduate students of speech therapy and audiology sciences at the University of Leuven completed an anonymous one-item questionnaire. I asked them to write down a good example of a large number that might be used for illustrative purposes. Thirty-three received such a request (factual representation condition). I asked sixteen to write down "a good example of a large number as people represent it in their imagination" (people's subjective representation condition), and I asked another sixteen to provide a good example of a large number as you represent it in your imagination (own subjective representation condition).

The results showed number sizes ranging from three to 26 digits, but failed to yield any evidence that participants would produce smaller numbers in the subjective representation conditions than in the factual representation condition. This counterevidence may be too limited to dismiss the shadow of reality hy- 
pothesis for good, but it provides possible alternatives such as the following pregnance hypothesis.

\section{The Pregnance Hypothesis}

The pregnance hypothesis is based on an eye-catching difference obtained between the above subjective representation and factual representation conditions. In the subjective representation condition, $76 \%$ of the numbers ended with zero, versus only $50 \%$ in the factual representation condition, $\chi^{2}(1, N=65)=$ $4.63, p<.02, E S=.27$. No less than $83 \%$ of these numbers consisted of a single digit and a long string of zeros. No difference was observed between conditions contrasting people's versus own subjective representations.

Apparently, the subjective representational status stimulated the production of round numbers, which may be a manifestation of the classic Gestalt psychological law of closure. In an analogous way, the weak belief effect, manifested by minimizing the difference in length between railroad-like lines, may fit the law of good shape. Both laws are variants of the basic law of pregnance (Ash, 1995). This suggests my hypothesis that awareness of the subjective status of a representation increases the degree to which the representation is shaped in accordance with the law of pregnance.

\section{Pilot Study 2: Exploring the Pregnance Hypothesis}

Because the law of pregnance originated from the psychology of figural perception (Ash, 1995), two female research assistants individually presented 235 first-year psychology undergraduate students (38\% males) from the University of Leuven with the simple request to draw a polygon. I implemented this task at the end of an experimental session that was unrelated to figural perception. The research assistants were unacquainted with the purpose of the drawing task. As in Study 1, the research assistants provided each student with a framed sheet. An accompanying text explained that the experimenter needed drawings to be used in a forthcoming investigation. The experimenters refreshed the students' basic school knowledge about polygons by introducing examples of polygons (e.g., triangles, quadrangles, pentagons)." For 114 participants, the instruction read: "Draw a polygon" (factual representation condition); for 121 participants it read: "Draw a polygon as you think that people represent a polygon in their imagination" (subjective representation condition). The research assistants debriefed the participants after the sessions were over.

On the basis of the pregnance hypothesis, one could expect more regular polygons in the subjective representation than in the factual representation condition. However, because the drawings were sketched, the polygons were irregular when enlarged and it was hard to separate genuine irregulars from poorly drawn regulars. A more reliable distinction could be made between conventional convex and pla- 
nar polygons on the one hand, and atypical figures with indentions, intersecting edges, or nonplanar coordinates on the other hand. Only $20 \%$ of the polygons belonged to the latter atypical category. In line with the pregnance hypothesis, there were slightly more atypical cases in the factual representation condition than in the subjective representation condition ( $21 \%$ vs. $18 \%)$, but the difference was not statistically significant and too small to provide any support to the hypothesis.

Because results regarding atypical cases were inconclusive, I restricted further analyses to the 189 conventional cases. Counting the number of sides or angular points, I easily classified them by the number of angles the appropriate type of polygon. This classification yielded some remarkable outcomes (consistent across gender groups, for which reason gender is further ignored).

An unanticipated outcome was that $71 \%$ of 90 participants who were asked to draw a polygon (factual representation condition) produced polygons with an even number of angles-for the most part quadrangles, hexagons and octagons. Only 29\% produced polygons with an odd number of angles - mostly triangles and pentagons. The difference was statistically significant, $\chi^{2}(1, N=90)=16.04, p<.00006$ (twotailed). Such a result suggests that an even number of angles may be a mark of good, or pregnant, form." If so, the pregnance hypothesis predicts that the preponderance of even-angled polygons would be even greater in the subjective representation condition. Consistent with the hypothesis, 99 participants produced $83 \%$ even-angled polygons versus only $17 \%$ odd-angled polygons. As in the factual representation conditions, the polygons ranged from triangles to octagons, but the contrast between even- and odd-angled polygons was significantly stronger in the subjective representation condition, $\chi^{2}(1, N=189)=3.68, p<.03$ (one-tailed), $E S=.14$.

\section{Generalizing the Pregnance Hypothesis}

The outcomes of two pilot studies urge future researchers to continue along the lines of the pregnance hypothesis. It is feasible that Hendrickx's (1997) participants sacrificed veridicality for the sake of local coherence. To achieve coherence, they may have relied on stereotypes associating tallness of stature with boastfulness or at least with an inflated physical self-image. In addition, the concept of pregnance should not be restricted to closure and good form. Pregnance has been associated with parsimony, regularity, symmetry, simplicity, and other related concepts (Ash, 1995). McKoon and Radcliff's (1992) local coherence may be added to this list. Hence, I end with two examples from the literature on evaluative meaning processing that argue for the pregnance hypothesis by highlighting the role of simplicity and symmetry.

\section{Simplicity}

There is a long tradition, reaching back to Wundt (1896), that stresses a simple, evaluativemeaning concept modeled as a single general good/bad dimension 
that reflects incentive values for approach and avoidance. However, since the pioneering study of Rosenberg, Nelson, and Vivekanathan (1968), students of social perception and cognition have handled a more complex model involving two evaluative good/bad dimensions, only one of which reflects incentive values for approach and avoidance (Peeters, 1986; Wentura, Rothermund, \& Bak, 2000). Cross-cultural validity has been claimed for the simple model (Osgood, May \& Miron, 1975) as well as for the complex model (White, 1980). Rosenberg and his colleagues also found that the unidimensional model, if completed with nonevaluative dimensions, could account nearly as well for the data available as did their preferred two-dimensional model. Apparently, people hold two models of evaluation: a simple and a complex one. In a recent study (Peeters, Cornelissen, \& Pandelaere, 2003), the authors unexpectedly found that perceivers relied on the more complex model only when they themselves decided whether to approach or avoid a target person. However, when anticipating the decision of another person, the perceivers assumed that the other would decide following the simple model. For instance, the two-dimensional model handles sociable and industrious as positive traits belonging to different evaluative dimensions. When anticipating their own possible behavior, perceivers anticipated approach behavior directed to the sociable person but not necessarily to the industrious person. However, when asked to anticipate another person's approach tendencies, the participants expected the other to approach targets irrespective of whether the other described them as industrious or as social. Thus, they assumed the other person would decide on the basis of a simple concept of evaluation, collapsing the two dimensions of the complex model into a single good/bad dimension.

\section{Symmetry}

Evaluative dimensions have often been conceived as symmetrical constructs contrasting positive and negative poles equidistant from a neutral middle. There is evidence that this symmetry does not reflect real experiences, which better fit an asymmetric model with the neutral middle nearer to the positive pole (see Peeters \& Czapinski, 1990; Rozin \& Royzman, 2001; Taylor, 1991). However, Wyer and Hinckle (1976) found that participants made evaluations consistent with the simpler symmetrical model when they did not report their own evaluations but evaluations they presumed another person would make.

\section{Conclusion}

In the light of the available evidence, the most promising hypothesis may relate the subjective status effect to an increased impact of the law of pregnance (e.g., tendencies towards closure, good shape, simplicity, symmetry). However, the alternative shadow of reality hypothesis has intuitive appeal and future researchers should take it into account. 


\section{REFERENCES}

Ash, M. G. (1995). Gestalt psychology in German culture 1890-1967: Holism and the quest for objectivity. Cambridge, England: Cambridge University Press.

Cohen, J. (1977). Statistical power analysis for the behavioral sciences. New York: Academic Press.

Frege, G. (1892). Sinn und Bedeutung [On sense and reference]. Zeitschrift für Philosophie und Philosophische Kritik, 100, 25-50.

Gerrig, R. J. (1989). Suspense in the absence of uncertainty. Journal of Memory and Language, 28, 633-648.

Gerrig, R. J. (1993). Experiencing narrative worlds: On the psychological activities of reading. New Haven, CT: Yale University Press.

Gerrig, R. J., \& Rapp, D. N. (2004). Psychological processes underlying literary impact. Poetics Today, 25, 265-281.

Gilbert, D. T. (1991). How mental systems believe. American Psychologist, 46, 107-119.

Gilbert, D. T., Tafarodi, R. W., \& Malone, P. S. (1993). You can't not believe everything you read. Journal of Personality and Social Psychology, 65, 221-233.

Grice, H. P. (1975). Logic and conversation. In P. Cole \& J. L. Morgan (Eds.), Syntax and semantics 3: Speech acts (pp. 41-58). San Diego, CA: Academic Press.

Heider, F. (1958). The psychology of interpersonal relations. New York: Wiley.

Hendrickx, A. (1997). Ambiguity: An integrated approach. In A. Sorace, C. Heycock, \& R. Shillcock (Eds.), Proceedings of the GALA 97 Conference on Language Acquisition (pp. 57-61). Edinburgh, Scotland: University of Edinburgh.

Hilton, D. J. (1990). Conversational processes and causal explanation. Psychological Bulletin, 107, 65-81.

Hilton, D. J. (1991). A conversational model of causal explanation. In W. Stroebe \& M. Hewstone (Eds.), European Review of Social Psychology (Vol. 4, pp. 113-139). Chichester, England: Wiley.

Hilton, D. J. (1995). The social context of reasoning: Conversational inference and rational judgment. Psychological Review, 118, 248-271.

Hoorens, V. (1993). Self-enhancement and superiority biases in social comparison. In W. Stroebe \& M. Hewstone (Eds.), European Review of Social Psychology (Vol. 4, pp. 113-139). Chichester, England: Wiley.

Kruglanski, A. W. (1989). The psychology of being "right": The pattern of accuracy in social perception and cognition. Psychological Bulletin, 106, 395-409.

Levinson, S. C. (2000). The theory of generalized conversational implicature. Cambridge, MA: MIT Press.

McKay, T. (2000). Propositional attitude reports. In E. N. Zalta (Ed.), The Stanford Encyclopedia of Philosophy (Spring 2000 ed.). Retrieved August 15, 2005, from Website: http://plato.stanford.edu/archives/spr2000/entries/prop-attitudes-reports

McKinsey, M. (1999). The semantics of belief ascriptions. Noûs, 33, 519-557.

McKoon, G., \& Radcliff, R. (1992). Inference during reading. Psychological Review, 99, 440-466.

Moore, C., \& Furrow, D. (1991). The development of language of belief: The expression of relative certainty. In D. Frye, \& C. Moore (Eds.), Children's theories of mind: Mental states and social understanding (pp. 173-193). Hillsdale, NJ: Erlbaum.

Osgood, C. E., May, W. H., \& Miron, M. S. (1975). Cross-cultural universals of affective meaning. Urbana: University of Illinois Press.

Peeters, G. (1977). Language, meaning, and cognition: A social-psychological approach. Communication and Cognition, 10, 63-88.

Peeters, G. (1986). Good and evil as softwares of the brain: On psychological "immediates" underlying the metaphysical "ultimates." A contribution from cognitive social 
psychology and semantic differential research. Ultimate Reality and Meaning: Interdisciplinary Studies in the Philosophy of Understanding, 9, 210-231.

Peeters, G., Cornelissen, I., \& Pandelaere, M. (2003). Approach-avoidance values of target-directed behaviors elicited by target-traits: The role of evaluative trait dimensions. Current Psychology Letters, 11(2). Retrieved May 20, 2005, from http://cpl.revues.org/document396.html

Peeters, G. \& Czapinski, J. (1990). Positive-negative asymmetry in evaluations: The distinction between affective and informational negativity effects. In W. Stroebe \& M. Hewstone (Eds.), European Review of Social Psychology (Vol. 1, pp. 33-60). Chichester, England: Wiley.

Rapp, D. N., \& Gerrig, R. J. (2002). Readers' reality-driven and plot-driven analyses in narrative comprehension. Memory and Cognition, 30, 779-788.

Rosenberg, S., Nelson, C., \& Vivekanathan, P. S. (1968). A multidimensional approach to the structure of personality impressions. Journal of Personality and Social Psychology, 9, 283-294.

Rozin, P., \& Royzman, E. B. (2001). Negativity bias, negativity dominance, and contagion. Personality and Social Psychology Review, 5, 296-320.

Russel, B. (1912). The problems of philosophy. Oxford, England: Oxford University Press.

Schwanenflugel, P. J., Fabricius, W. V., \& Noyes, C. R. (1996). Developing organization of mental verbs: Evidence for the development of a constructivist theory of mind in middle childhood. Cognitive Development, 11, 265-294.

Schwanenflugel, P. J., Fabricius, W. V., Noyes, C. R., Bigler, K. D., \& Alexander, J. M. (1994). The organization of mental verbs and folk theories of knowing. Journal of Memory and Language, 33, 376-395.

Schwanenflugel, P. J., Martin, M., \& Takahashi, T. (1999). The organization of verbs of knowing: Evidence for cultural commonality and variation in theory of mind. Memory and Cognition, 27, 813-825.

Schwarz, N. (1996). Cognition and communication: judgmental biases, research methods, and the logic of conversation. Hillsdale, $\mathrm{NJ}$ : Erlbaum.

Schwarz, N. (1998). Warmer and more social: Recent developments in cognitive social psychology. Annual Review of Sociology, 24, 239-264.

Taylor, S. E. (1991). Asymmetrical effects of positive and negative events: The mobilization-minimization hypothesis. Psychological Bulletin, 110, 67-85.

Wentura, D., Rothermund, K., \& Bak, P. (2000.) Automatic vigilance: The attention-grabbing power of approach- and avoidance-related social information. Journal of Personality and Social Psychology, 78, 1024-1037.

White, G. M. (1980). Conceptual universals in interpersonal language. American Anthropologist, 82, 759-781.

Wolf, F. M. (1986). Meta-analysis: Quantitative methods for research synthesis. Beverly Hills, CA: Sage.

Wundt, W. (1896). Grundriss der Psychologie (6th ed.) [Outlines of Psychology]. Leipzig, Germany: Engelman.

Wyer, R. S., \& Hinckle, R. L. (1976). Informational factors underlying inferences about hypothetical persons. Journal of Personality and Social Psychology, 34, 481-495.

Yates, J. (1985). The content of awareness is a model of the world. Psychological Review, 92, 249-284. 


\section{APPENDIX A \\ (Forms Translated From Dutch)}

\section{Studies 1 and 2}

Complete or mark: Date

Gender: male/female

\section{THE OPTIMAL COMBINATION OF PICTURES AND TEXT}

We are investigating when illustrations or pictures, which are inserted in a text, are experienced as going well with the text. The simple task that follows is only one of many simple partial tasks.

Below there is a frame with a caption.

We ask you to draw two straight lines within the frame. You draw them so that you have the feeling that the drawing and the caption fit well together.

We ask for straight lines, no curves, but they can be freely sketched. They can be horizontal, vertical, or diagonal. What really matters is that we see clearly where each line begins and where it ends. And we should be able, of course, to distinguish between an upper line and a lower line.

Note that this task takes no more than one or two minutes. We do not expect that you make a long reflection but that you follow your intuition. Do not glance at lines others may be drawing, for they have different tasks with different captions.

Many thanks for your help. For more information about this research, please contact me in room 3.10 of the faculty.

Guido Peeters

\section{Study 3}

Complete or mark: Date

Gender: male/female

\section{RESEARCH PROJECT ON PERCEPTION, IMAGINATION, AND MEMORY}

We ask for your help with the preparation of a research project on perception, imagination, and memory. We need simple drawings. Therefore, we ask you what follows.

We ask for straight lines, no curves, but they can be freely sketched. They can be horizontal, vertical, or diagonal. What really matters is that we see clearly where each line begins and where it ends. We should be able, of course, to distinguish between an upper line and a lower line.

Note that this task takes no more than one or two minutes. We do not expect that you make a long reflection but that you follow your intuition. Do not glance at lines others may be drawing, for they have different instructions.

Thanks for your help. For more information about this research, please contact me in room 3.10 of the faculty.

Guido Peeters 


\section{APPENDIX B \\ Text Varying Across Conditions}

\section{Study 1: Captions Used to Implement Conditions C1-C16 \\ Assertion Conditions \\ C1 The upper line is longer than the lower line. \\ C2 The upper line is surely longer than the lower line. \\ C3 It is sure that the upper line is longer than the lower line.}

\section{Covert Belief Conditions}

C4 I think the upper line is longer than the lower line.

C5 I am sure the upper line is longer than the lower line.

C6 I see the upper line is longer than the lower line.

C7 Kris thinks the upper line is longer than the lower line.

C8 Kris is sure the upper line is longer than the lower line.

C9 Kris sees the upper line is longer than the lower line.

C10 Everybody thinks the upper line is longer than the lower line.

C11 Everybody is sure the upper line is longer than the lower line.

C12 Everybody sees the upper line is longer than the lower line.

\section{Overt Belief Conditions}

C13 Kris says the upper line is longer than the lower line.

C14 Kris says: "The upper line is longer than the lower line."

C15 Everybody says the upper line is longer than the lower line.

C16 Everybody says: "The upper line is longer than the lower line."

\section{Study 2: Captions Used to Implement Conditions C17-C20 \\ Assertion Condition}

C17 The upper line is longer than the lower line.

\section{Belief Conditions}

C18 Kris says the upper line is longer than the lower line.

C19 Everybody says the upper line is longer than the lower line.

C20 Like everybody else, Kris says that the upper line is longer than the lower line.

\section{Study 3: Variable Text Fragments Used in Conditions C17-C20 Factual Representation Condition}

C21 First Text Block: Draw two straight lines that fit the following description: "The upper line is longer than the lower line."

Second Text Block: Your task is to draw two lines of which the upper line is longer than the lower line.

\section{Subjective Representation Conditions}

C22 First Text Block: Draw two straight lines as people represent them in their imagination when they get the following description: "The upper line is longer than the lower line." Draw these lines in the frame below as people represent them in their imagination.

Second Text Block: Your task is to draw how people represent in their imagination two lines of which the upper line is longer than the lower line. 


\section{APPENDIX B-(Continued)}

C23 First Text Block: Draw two straight lines as you think that people represent them in their imagination when they get the following description: "The upper line is longer than the lower line." Draw these lines in the frame below as you think that people represent them in their imagination.

Second Text Block: Your task is to draw how people represent in their imagination two lines of which the upper line is longer than the lower line.

C24 First Text Block: Draw two straight lines as you represent them in your imagination and that fit the following description: "The upper line is longer than the lower line." Draw these lines in the frame below as you represent them in your imagination.

Second Text Block: Your task is to draw how you represent in your imagination two lines of which the upper line is longer than the lower line.

C25 First Text Block: Draw two straight lines as you think that you represent them in your imagination and that fit the following description: "The upper line is longer than the lower line." Draw these lines in the frame below as you think that you represent them in your imagination.

Second Text Block: Your task is to draw how you think you represent in your imagination two lines of which the upper line is longer than the lower line. 
Copyright of Genetic, Social \& General Psychology Monographs is the property of Heldref Publications and its content may not be copied or emailed to multiple sites or posted to a listserv without the copyright holder's express written permission. However, users may print, download, or email articles for individual use. 